\title{
Association between weight control failure and suicidal ideation in overweight and obese adults: a cross-sectional study
}

\author{
Yeong Jun Ju, ${ }^{1,2}$, Kyu-Tae Han ${ }^{1,2}$, Tae-Hoon Lee ${ }^{1,2}$, Woorim Kim ${ }^{1,2}$, Jeong Hun Park ${ }^{4}$ and Eun-Cheol Park ${ }^{2,3,5^{*}}$
}

\begin{abstract}
Background: Korea has the highest suicide rate in the OECD and is one of the few OECD countries whose suicide rates have not decreased in recent years. To address these issues, we investigated the effects of weight control failure on suicidal ideation in the overweight and obese populations.

Methods: We performed a cross-sectional study using data from the Korea National Health and Nutrition Examination Survey (2008-2012) consisting of 6621 individuals 40 years of age or older. Logistic regression analysis was used to identify the relationship between weight control failure and suicidal ideation in the overweight and obese populations.

Results: A total of 6621 participants were analyzed in this study (overweight group: 2439; obese group: 4182). Among them, weight control failure (weight gain with weight loss efforts) was experienced in 962 obese (males $16.3 \%$, females $29.6 \%$ ) and 412 overweight individuals (males $9.1 \%$, females $23.4 \%$ ). Weight control failure was significantly associated with suicidal ideation in obese females $(\mathrm{OR}=1.70,95 \% \mathrm{Cl} 1.21-2.39)$, but this association was not significant in obese males or in either sex of the overweight group.

Conclusions: Findings from this study suggest that weight control failure is associated with an increased risk of suicidal ideation among obese women. Furthermore, intervention programs that aim to address the prevalence of suicide, especially for obese women, are needed.
\end{abstract}

Keywords: Suicidal ideation, Obesity, Weight control failure, Weight stigma, Middle age

\section{Background}

Suicide is becoming a public health issue in many countries, and even more so in Korea [1]. South Korea's suicide rate has been the highest among OECD countries for over a decade. In 2013, the rate of death by intentional self-harm in South Korea was 29.1/100,000 individuals, which was followed by the rates in Japan, Hungary, and Slovenia at nearly 20/100,000 individuals; the OECD average rate was $12 / 100,000$ individuals [2]. Meanwhile, one previous study reported that suicidal ideation is the main thought process leading to suicide [3]. Those who do not envision suicide rarely attempt suicide, whereas $34-42 \%$ of individuals who imagine

\footnotetext{
* Correspondence: ecpark@yuhs.ac

${ }^{2}$ Institute of Health Services Research, Yonsei University College of Medicine, Seoul, Republic of Korea

${ }^{3}$ Department of Preventive Medicine, Yonsei University College of Medicine, Seoul, Republic of Korea

Full list of author information is available at the end of the article
}

committing suicide subsequently attempt it [4]. Hence, it is necessary to approach suicide prevention in terms of prevention and identification of the factors that influence suicidal ideation.

Previous studies to clarify the factors affecting suicidal ideation have generally considered relevant health behaviors (drinking, socioeconomic conditions, internet addiction) and mental health conditions (depression, mood disorders, anxiety) [5-7]. However, there remain many issues and debates related to mental health, and suicidal problems in particular, in South Korea. Therefore, we determined it necessary to address suicidal problems from another perspective. In particular, we focused on suicidal problems related to weight control issues in the overweight and obese population of South Korea. However, few studies have examined how weight status impacts suicidal ideation. 
Weight status has been classified as underweight, normal weight, overweight or obese. According to a previous study, weight status was associated with major depression, suicide attempts, and suicidal ideation [3]. Obesity is an especially important factor affecting mental health [8,9]; obese populations have a higher rate of depression and suicidal ideation than that of the normal weight population [10]. In addition, obesity has been associated with increased morbidity and mortality risks $[11,12]$. For that reason, many obese individuals attempt to lose weight, and weight reduction is an important aspect of obesity treatment [13]. As a result, weight control is an important issue for the obese population. Given the relationship between obesity and mental health, a study on whether weight control influences suicidal ideation is needed.

Of course, previous studies on suicidal ideation related to weight control exist. However, those studies only focused on adolescents [14-16], while the present study focuses on middle-aged adults. The study was limited to middle-age subjects (40 years or older), because the incidence of many health problems tends to increase during this period [17]. Also, weight status and mental health problems may be different in middle-aged and older adults compared with younger adults, because functional limitations and medical comorbidities related to aging may lead to weight change and mood changes [18]. Especially, women usually experienced the menopause in middle-age, which may leads to health risks such as weight gain $[19,20]$. We analyzed the effects of weight control failure on suicidal ideation in the overweight and obese populations and examined the relationship between weigh control failure and suicidal ideation according to income level, household composition, and menopause status.

\section{Methods}

\section{Study population}

For this cross-sectional study, we used raw data from the Korea National Health and Nutrition Examination Surveys (KNHANES) conducted in 2008-2012 (4th and 5th KNHANES); these are cross-sectional surveys that have been conducted annually by the Korea Centers for Disease Control and Prevention (KCDC). These are cross-sectional surveys with study populations from multistage, stratified area probability samples of civilian non-institutionalized Korean households by geographic area, age, and gender groups. This survey is composed of three parts-a health interview, health examination, and nutrition survey-all of which were performed by trained medical staff and dieticians. We used data from all three parts. These data were collected from a total of 45,811 participants during 2008-2012 (8058 in 2008, 8518 in 2009, 8958 in 2010, 10,533 in 2011, 9744 in 2012). The overall response rates were $74.3 \%$ in 2008,
$79.2 \%$ in $2009,81.9 \%$ in $2010,80.4 \%$ in $2011,80.8 \%$ in 2012. In our analysis, the subjects were divided into two subgroups according to BMI: $23-24.9 \mathrm{~kg} / \mathrm{m}^{2}$ (overweight) or $\geqq 25 \mathrm{~kg} / \mathrm{m}^{2}$ (obese). Any respondents who did not provide data on BMI, suicidal ideation, education level, income, household composition, marital status, moderate physical activity, stress awareness, alcohol consumption, or who were under the age of 40 years were excluded from the study (see details in Fig. 1). In addition, we excluded those who did not undergo weight control attempts or who attempted to gain weight to investigate the success or failure of weight control efforts in the participants. A total of 6621 eligible participants were included in the present study (2439 overweight; 4182 obese). Meanwhile, considering that sex affects mental health, the present study analyzed men and women separately (overweight: 1116 males, 1323 females; obese: 2081 males, 2101 females) [4, 21]. The KNHANES data are openly available at the KNHANES website: https://knhanes.cdc.go.kr/knhanes/eng/index.do.

Ethical approval for this study was granted by the institutional review board (IRB) of the KCDC Seoul, Korea (IRB \#: 2008-04EXP-01-C; 2009-01CON-03-2C; 201002CON-21-C; 2011-02CON-06-C; 2012-01EXP-01-2C).

\section{Measures \\ Suicidal ideation}

We used data from a self-reported questionnaire, in which suicidal ideation was assessed by a single item: "Have you ever seriously thought about suicide in the past year?" Responses were rated on a 2-point scale: yes or no. Despite limitations due to the question's simplicity, previous studies have successfully used this method based on KNHANES data [4, 22, 23].

\section{Weight control failure}

To assess the success or failure of weight control efforts, we used data from a self-reported questionnaire regarding changes in body weight and weight control efforts. Change in body weight was assessed by the single question, "Have you experienced a change in body weight in comparison with the last year?" Responses were rated on a 3-point scale: weight maintenance, weight gain, or weight loss. Weight gain is composed of three parts: increases of $3-$ $6 \mathrm{~kg}, 6-10 \mathrm{~kg}$, or $10 \mathrm{~kg}$ or more. Weight loss was composed of three parts: decreases of 3-6 kg, 6-10 kg, or $10 \mathrm{~kg}$ or more. Weight control efforts were assessed by the single question, "Have you ever tried to control your weight voluntarily in the last year?" Responses were rated on a 4-point scale: made efforts for weight loss, made efforts for weight maintenance, made efforts for weight gain, did not make efforts for weight control. Finally, we classified weight control failure as "yes" or "no". Those who experienced weight gain during weight loss efforts 


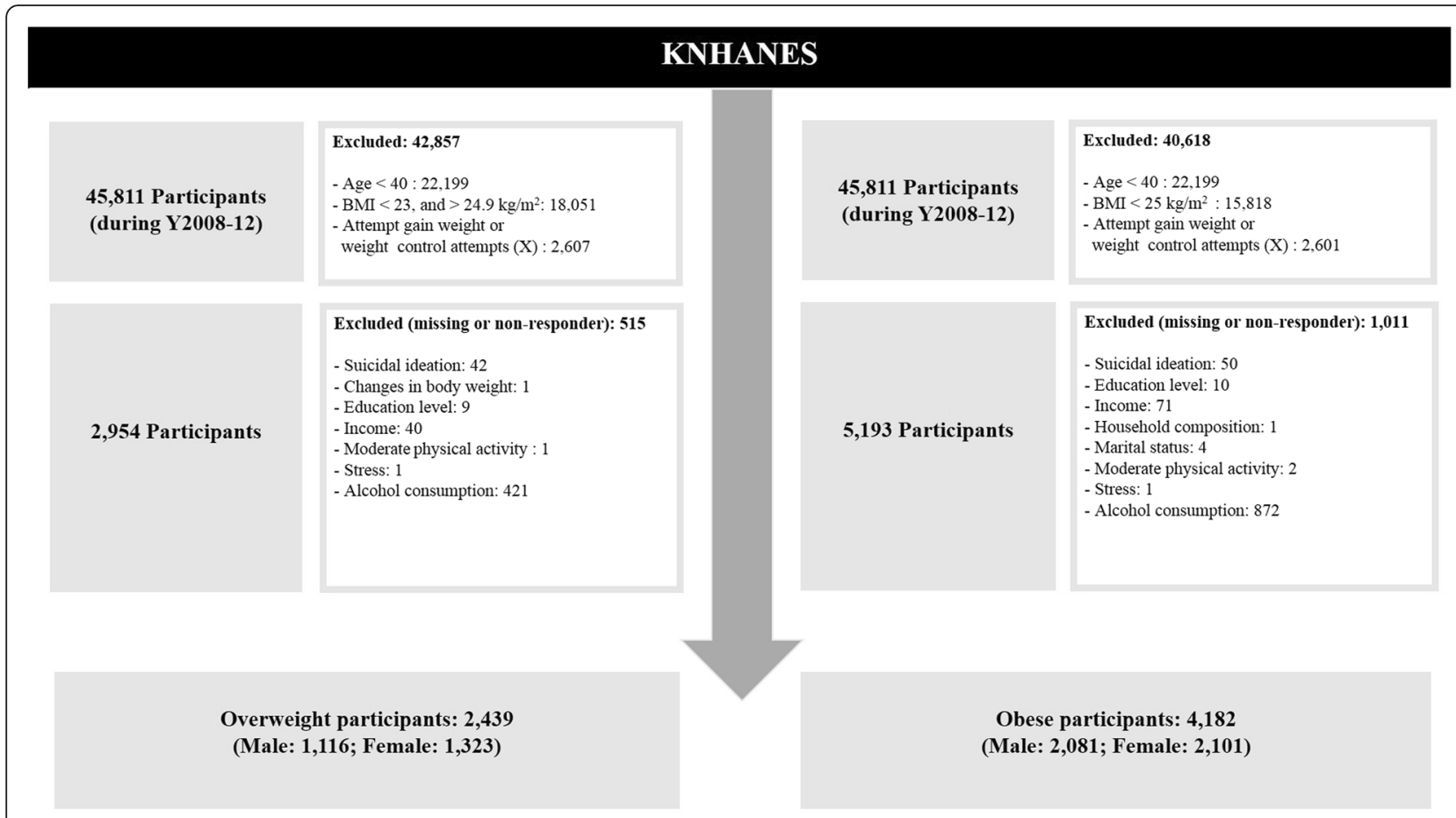

Fig. 1 Flow diagram of the study participants

were categorized as "yes". Those who experienced weight loss during weight loss efforts or weight maintenance during weight maintenance efforts were classified as "no".

\section{Criteria of obesity}

The World Health Organization Regional Office for the Western Pacific Region recommends defining obesity in Asians as those with a BMI $\geqq 25 \mathrm{~kg} / \mathrm{m}^{2}$. Korea officially uses this definition when calculating the prevalence of obesity in Korea. Overweight in Asians is defined as a BMI of $23-24.9 \mathrm{~kg} / \mathrm{m}^{2}$ [24]. Therefore, our study used this definition.

\section{Covariates}

We included age, sex, income, educational level, economic activity, marital status, household composition, smoking status, alcohol consumption, stress awareness, depressive symptoms, perceived body image, perceived health status, moderate physical activity, menopause (only for females) and survey year as covariates. Age was categorized into four groups, starting with 40 years of age. Income status was classified as "low", "middle", or "high". Economic activity was classified as "employed" or "unemployed". Household composition was assessed by the question "What is your household composition?" This variable was categorized as "one-generation household" (single-person household), "two-generation household" (couple + children, single parent + children, couple + parents, couple + single parent, grandparents + grandchildren, single grandparent + grandchildren), and "three-generation household" (couple + parents + children, couple + single parent + children). Stress awareness was classified as "high" or "low". Depressive mood was assessed by duration of the depressive mood during the past 2 weeks (present, absent). Subjective body perception was assessed by the question "How would you categorize your current body shape?" (very thin, slightly thin, normal, slightly fat, or very fat). We categorized this as "slim and normal" (very thin, slightly thin, or normal) or "fat" (slightly fat or very fat). Moderate physical activity was classified as whether respondents performed moderate physical activity for 30 min per session more than five times per week. Menopause status was assessed as "yes" (experienced menopause) or "no" (not applicable or yet to experience menopause).

\section{Statistical analysis}

We first examined the distribution of each categorical variable. The Chi-squared test was used to calculate the frequencies and percentages of the variables and to identify significant differences between groups. The analyses for incidence of suicidal ideation by sex were also performed. In addition, to produce an unbiased national estimate, a sample weight was assigned for the participating individuals to represent the Korean population. The sampling weight were calculated by accounting for the complex survey design, survey nonresponse, and poststratification [25]. Next, multivariable logistic regression 
Table 1 General characteristics of participants according to suicidal ideation in male

\begin{tabular}{|c|c|c|c|c|c|c|}
\hline \multirow[t]{4}{*}{ Variables } & \multicolumn{6}{|c|}{ Suicidal ideation (Male) } \\
\hline & \multicolumn{3}{|c|}{ BMI $23 \sim 24.9$} & \multicolumn{3}{|l|}{$\mathrm{BMI} \geq 25$} \\
\hline & Total & Yes & & Total & Yes & \\
\hline & N (\%) & N (\%) & $P$-value* & N (\%) & N (\%) & $P$-value* \\
\hline Weight control failure & & & 0.7918 & & & 0.6632 \\
\hline No & $1014(90.9)$ & $101(10.0)$ & & $1741(83.7)$ & $161(9.3)$ & \\
\hline Yes & $102(9.1)$ & $11(10.8)$ & & $340(16.3)$ & $34(10.0)$ & \\
\hline Age & & & 0.7125 & & & 0.0053 \\
\hline $40 \sim 49$ & $335(30.0)$ & $29(8.6)$ & & $760(36.5)$ & $56(7.4)$ & \\
\hline $50 \sim 59$ & $365(32.7)$ & $40(10.9)$ & & $612(29.4)$ & $59(9.6)$ & \\
\hline $60 \sim 69$ & $276(24.7)$ & $27(9.8)$ & & $478(23.0)$ & $45(9.4)$ & \\
\hline$\geq 70$ & $140(12.6)$ & $16(11.4)$ & & $231(11.1)$ & $35(15.1)$ & \\
\hline Education level & & & 0.0523 & & & $<.0001$ \\
\hline Less than middle school & $346(31.0)$ & $46(13.3)$ & & $659(31.7)$ & $91(13.8)$ & \\
\hline High school graduate & $368(33.0)$ & $32(8.7)$ & & $697(33.5)$ & $62(8.9)$ & \\
\hline University graduate & $402(36.0)$ & $34(8.5)$ & & $725(34.8)$ & $42(5.8)$ & \\
\hline Income & & & 0.0021 & & & $<.0001$ \\
\hline High & $370(33.2)$ & $23(6.2)$ & & $643(30.9)$ & $40(6.2)$ & \\
\hline Middle & $535(47.9)$ & $57(10.6)$ & & $1030(49.5)$ & $89(8.6)$ & \\
\hline Low & $211(18.9)$ & $32(15.2)$ & & $408(19.6)$ & $66(16.2)$ & \\
\hline Economic activity & & & 0.0035 & & & 0.0012 \\
\hline Employed & $843(75.5)$ & $72(8.5)$ & & $1658(79.7)$ & $138(8.3)$ & \\
\hline Unemployed & $273(24.5)$ & $40(14.6)$ & & $423(20.3)$ & $57(13.5)$ & \\
\hline Marital status & & & 0.9428 & & & 0.0297 \\
\hline Married & $1097(98.3)$ & $110(10.0)$ & & $2039(98.0)$ & $187(9.2)$ & \\
\hline Single & $19(1.7)$ & $2(10.5)$ & & $42(2.0)$ & $8(19.0)$ & \\
\hline Household composition & & & 0.0936 & & & 0.1951 \\
\hline First Generation & $354(31.7)$ & $34(9.6)$ & & $635(30.5)$ & $67(10.5)$ & \\
\hline Two Generation & $624(55.9)$ & $57(9.1)$ & & $1203(57.8)$ & $112(9.31)$ & \\
\hline Three Generation & $138(12.4)$ & $21(15.2)$ & & $243(11.7)$ & $16(6.6)$ & \\
\hline Stress awareness & & & $<.0001$ & & & $<.0001$ \\
\hline Low & 885 (79.3) & $51(5.8)$ & & $1610(77.4)$ & $92(5.7)$ & \\
\hline High & $231(20.7)$ & $61(26.4)$ & & $471(22.6)$ & $103(21.9)$ & \\
\hline Depression mood & & & $<.0001$ & & & $<.0001$ \\
\hline Absent & $1015(91.0)$ & $60(5.9)$ & & $1895(91.1)$ & $105(5.5)$ & \\
\hline Present & $101(9.0)$ & $52(51.5)$ & & $186(8.9)$ & $90(48.4)$ & \\
\hline Alcohol intake & & & 0.4252 & & & 0.2811 \\
\hline No & $152(13.6)$ & $18(11.8)$ & & $259(12.5)$ & $29(11.2)$ & \\
\hline Yes & $964(86.4)$ & $94(9.7)$ & & $1822(87.5)$ & $166(9.1)$ & \\
\hline Moderate physical activity & & & 0.3373 & & & 0.1114 \\
\hline No & $586(52.5)$ & $54(9.2)$ & & $1061(51.0)$ & $110(10.4)$ & \\
\hline Yes & $530(47.5)$ & $58(10.9)$ & & $1020(49.0)$ & $85(8.3)$ & \\
\hline Subjective body perception & & & 0.6795 & & & 0.2492 \\
\hline Slim \& Normal & $737(66.0)$ & $72(9.8)$ & & $425(20.4)$ & $46(10.8)$ & \\
\hline
\end{tabular}


Table 1 General characteristics of participants according to suicidal ideation in male (Continued)

\begin{tabular}{|c|c|c|c|c|c|c|}
\hline Fat & $379(34.0)$ & $40(10.5)$ & & 1656 (79.6) & $149(9.0)$ & \\
\hline Perceive health status & & & $<.0001$ & & & $<.0001$ \\
\hline Healthy & $951(85.2)$ & $80(8.4)$ & & $1750(84.1)$ & $118(6.7)$ & \\
\hline Unhealthy & $165(14.8)$ & $32(19.4)$ & & 331 (15.9) & $77(23.3)$ & \\
\hline Year & & & 0.0942 & & & 0.1057 \\
\hline 2008 & $187(16.8)$ & $19(10.2)$ & & $364(17.5)$ & $42(11.5)$ & \\
\hline 2009 & $254(22.8)$ & 35 (13.8) & & $483(23.2)$ & $32(6.6)$ & \\
\hline 2010 & $203(18.2)$ & $14(6.9)$ & & $414(19.9)$ & $38(9.2)$ & \\
\hline 2011 & $236(21.1)$ & $26(11.0)$ & & $438(21.0)$ & $48(11.0)$ & \\
\hline 2012 & $236(21.1)$ & $18(7.6)$ & & $382(18.4)$ & $35(9.2)$ & \\
\hline Total & $1116(100.0)$ & $112(10.0)$ & & $2081(100.0)$ & $195(9.4)$ & \\
\hline
\end{tabular}

Notes: * P-values calculated by the chi-square test

analysis was used to examine the association between weight control failure and suicidal ideation while controlling for potential confounding variables such as age, sex, income, educational level, economic activity, marital status, household composition, alcohol consumption, stress awareness, depression mood, subjective body image, perceive health status, moderate physical activity, menopause (only females) and survey year. In addition, the fit of the model was assessed using the Hosmer-Lemeshow goodness-of-fit test; well-fitting logistic models have a nonsignificant goodness-of-fit [26]. Multicollinearity was tested using a variance inflation factor (VIF), which provided an index to measure how much the variance of an estimated regression coefficient increased due to collinearity. If the value of the variance inflation factor exceeded 10, a model was regarded as indicating collinearity. Finally, subgroup analyses were performed to evaluate the association between weight control failure and suicidal ideation according to income, household composition, or menopause. All of the analyses were performed using SAS 9.4 software (SAS Institute, Cary, NC, USA) and the statistical significance level was set at $p$-value $<0.05$.

\section{Results}

In our study, a total of 6621 participants were included to assess the association between weight control failure and suicidal ideation (overweight: 2439; obese: 4182). Tables 1 and 2 show the characteristics of the study population by sex. Among the obese population, suicidal ideation was higher in females than males. Suicidal ideation was noted in $195(9.4 \%)$ males and 432 (20.6 \%) females. Similar trends were demonstrated in the overweight population (10.0 \% in males, $17.5 \%$ in females). Weight control failure was more frequent in the obese group. Among the obese, weight control failure was higher in females than males (16.3 \% in males, $29.6 \%$ in females). Among the overweight, $9.1 \%$ of males versus $23.4 \%$ of females reported that they experienced weight control failure.
Notably, subjects in both the obese and overweight populations who experienced weight control failure were more likely to report suicidal thoughts. Regarding subjective body perception, among overweight males, 379 (34.0\%) reported being fat, whereas obese males reported being fat ( $n=1656 ; 79.6 \%$ ). Conversely, most females responded as being fat weight regardless of weight status $(64.7 \%$ in overweight, $88.9 \%$ in obese).

Table 3 shows the results of the logistic regression analysis for the association between weight control failure and suicidal ideation after multivariable adjustment. Weight control failure was significantly associated with suicidal ideation in the obese females. Among obese females, those with weight control failure were at 1.70-fold higher risk of suicidal ideation compared with those with weight control success or maintenance (OR $=1.70,95 \%$ CI 1.21-2.39), but this association was not significant in males of the obese or overweight group. In addition, among obese females, those with a low-income status were at a 2.11 -fold higher risk of suicidal ideation compared with those with a mid- to high-income status ( $\mathrm{OR}=2.12,95 \%$ CI 1.35-3.32); similar trends were evident in obese males $(\mathrm{OR}=1.79$, $95 \%$ CI 1.02-3.14). Obese females at menopause were at a 1.65-fold higher risk of suicidal ideation compared with obese females who had not yet reached menopause $(\mathrm{OR}=1.65,95 \%$ CI 1.02-2.65), whereas this association was not significant in overweight females. Stress awareness and depression mood were associated with suicidal ideation among all participants. The Hosmer-Lemeshow test was non-significant $(P=0.4263$ in overweight male group, $P=0.3487$ in overweight female group, $P=0.3573$ obese male group and $P=0.4730$ in obese female group) indicating adequate goodness-of-fit. In addition, All VIFs are below 2, indicating a lack of multicollinearity. The maximum VIF identified in our models was 1.83 (for obese male - age group).

The subgroup analysis results are shown in Table 4 . Subgroup analysis showed significant differences in each 
Table 2 General characteristics of participants according to suicidal ideation in female

\begin{tabular}{|c|c|c|c|c|c|c|}
\hline \multirow[t]{4}{*}{ Variables } & \multicolumn{6}{|c|}{ Suicidal ideation (Female) } \\
\hline & \multicolumn{3}{|c|}{ BMI $23 \sim 24.9$} & \multicolumn{3}{|l|}{$\mathrm{BMI} \geq 25$} \\
\hline & Total & Yes & & Total & Yes & \\
\hline & N (\%) & N (\%) & $P$-value* & N (\%) & N (\%) & $P$-value* \\
\hline Weight control failure & & & 0.1292 & & & 0.0239 \\
\hline No & $1013(76.6)$ & 168 (16.6) & & 1479 (70.4) & $285(19.3)$ & \\
\hline Yes & $310(23.4)$ & $63(20.3)$ & & $622(29.6)$ & $147(23.6)$ & \\
\hline Age & & & 0.1454 & & & $<.0001$ \\
\hline $40 \sim 49$ & $523(39.5)$ & $76(14.5)$ & & $648(30.8)$ & $107(16.5)$ & \\
\hline $50 \sim 59$ & $472(35.7)$ & $92(19.5)$ & & $696(33.1)$ & $127(18.2)$ & \\
\hline $60 \sim 69$ & $267(20.2)$ & $50(18.7)$ & & $530(25.2)$ & $32(24.9)$ & \\
\hline$\geq 70$ & $61(4.6)$ & $13(21.3)$ & & $227(10.9)$ & $66(29.1)$ & \\
\hline Education level & & & $<.0001$ & & & $<.0001$ \\
\hline Less than middle school & $613(46.3)$ & $138(22.5)$ & & $1275(60.7)$ & $310(24.3)$ & \\
\hline High school graduate & $487(36.8)$ & $68(14.0)$ & & $613(29.2)$ & $90(14.7)$ & \\
\hline University graduate & $223(16.9)$ & $25(11.2)$ & & $213(10.1)$ & $32(15.0)$ & \\
\hline Income & & & $<.0001$ & & & $<.0001$ \\
\hline High & $393(29.7)$ & $52(13.2)$ & & $479(22.8)$ & $74(15.4)$ & \\
\hline Middle & $662(50.0)$ & $106(16.0)$ & & $1112(52.9)$ & $221(19.9)$ & \\
\hline Low & $268(20.3)$ & $73(27.2)$ & & $510(24.3)$ & $137(26.9)$ & \\
\hline Economic activity & & & 0.4947 & & & 0.0074 \\
\hline Employed & $657(49.7)$ & $110(16.7)$ & & $1030(49.0)$ & $187(18.2)$ & \\
\hline Unemployed & $666(50.3)$ & $121(18.2)$ & & $1071(51.0)$ & $245(22.9)$ & \\
\hline Marital status & & & 0.5919 & & & 0.6217 \\
\hline Married & $1310(99.0)$ & $228(17.4)$ & & $2081(99.0)$ & $427(20.5)$ & \\
\hline Single & $13(1.0)$ & $3(23.1)$ & & $20(1.0)$ & $5(25.0)$ & \\
\hline Household composition & & & 0.0925 & & & 0.0049 \\
\hline First Generation & 391 (29.5) & $82(21.0)$ & & $750(35.7)$ & $169(22.5)$ & \\
\hline Two Generation & 779 (58.9) & $125(16.0)$ & & $1045(49.7)$ & $186(17.8)$ & \\
\hline Three Generation & $153(11.6)$ & $24(16.0)$ & & $306(14.6)$ & $77(25.2)$ & \\
\hline Stress awareness & & & $<.0001$ & & & $<.0001$ \\
\hline Low & $996(75.3)$ & $96(9.6)$ & & $1503(71.5)$ & $179(11.9)$ & \\
\hline High & $327(24.7)$ & $135(41.3)$ & & $598(28.5)$ & $253(42.3)$ & \\
\hline Depression mood & & & $<.0001$ & & & $<.0001$ \\
\hline Absent & $1091(82.5)$ & $108(9.90)$ & & $1691(80.5)$ & $203(12.0)$ & \\
\hline Present & $232(17.5)$ & $123(53.0)$ & & $410(19.5)$ & $229(56.0)$ & \\
\hline Alcohol intake & & & 0.7716 & & & 0.1623 \\
\hline No & $294(22.2)$ & $53(18.0)$ & & $468(22.3)$ & $107(22.9)$ & \\
\hline Yes & $1029(77.8)$ & $178(17.3)$ & & $1633(77.7)$ & $325(19.9)$ & \\
\hline Moderate physical activity & & & 0.6327 & & & 0.8825 \\
\hline No & 789 (59.6) & $141(17.9)$ & & $1271(60.5)$ & $260(20.5)$ & \\
\hline Yes & $534(40.4)$ & $90(16.9)$ & & $830(39.5)$ & $172(20.7)$ & \\
\hline Subjective body perception & & & 0.1517 & & & 0.3125 \\
\hline Slim \& Normal & $467(35.3)$ & $91(19.5)$ & & $234(11.1)$ & $54(23.1)$ & \\
\hline
\end{tabular}


Table 2 General characteristics of participants according to suicidal ideation in female (Continued)

\begin{tabular}{|c|c|c|c|c|c|c|}
\hline Fat & $856(64.7)$ & $140(16.4)$ & & $1867(88.9)$ & $378(20.3)$ & \\
\hline Perceive health status & & & $<.0001$ & & & $<.0001$ \\
\hline Healthy & $1052(79.5)$ & $135(12.8)$ & & $1473(70.0)$ & $226(15.3)$ & \\
\hline Unhealthy & $271(20.5)$ & $96(35.4)$ & & $628(30.0)$ & $206(32.8)$ & \\
\hline Menopause & & & 0.0891 & & & 0.0008 \\
\hline Not yet & $588(44.4)$ & $91(15.5)$ & & 793 (37.7) & $133(16.8)$ & \\
\hline Yes & 735 (55.6) & $140(19.0)$ & & $1308(62.3)$ & $299(22.9)$ & \\
\hline Year & & & 0.0625 & & & 0.0014 \\
\hline 2008 & $246(18.6)$ & $42(17.1)$ & & $384(18.3)$ & $92(24.0)$ & \\
\hline 2009 & $285(21.5)$ & $59(20.7)$ & & $476(22.7)$ & $115(24.2)$ & \\
\hline 2010 & 258 (19.5) & $48(18.6)$ & & $383(18.2)$ & $77(20.1)$ & \\
\hline 2011 & $268(20.3)$ & $42(15.7)$ & & $436(20.8)$ & 76 (17.4) & \\
\hline 2012 & $266(20.1)$ & $40(15.0)$ & & $422(20.1)$ & $72(17.1)$ & \\
\hline Total & $1323(100.0)$ & $231(17.5)$ & & $2101(100.0)$ & 432 (20.6) & \\
\hline
\end{tabular}

Notes: * $P$-values calculated by the chi-square test

group, even though modifying effects were not significant in the tests for interaction. Among obese males and females, those who had experienced weight control failure showed a trend towards a greater magnitude of suicidal ideation if they were of low, but not high, income status. Among obese females, there was a trend towards a greater magnitude of suicidal ideation if they lived on their own or in a two-generation household, but not if they lived with more family members. Among obese females experiencing menopause, those who had experienced weight control failure showed a trend towards a greater magnitude of suicidal ideation.

\section{Discussion}

South Korea currently has many public health issues. Among OECD members, Korea has ranked first in suicide rate for 11 years. The mortality rate from suicide increased rapidly from $22.6 \%$ in 2003 to $28.5 \%$ in 2013, making suicide the fourth leading cause of death in Korea [27]. In addition, the prevalence of obesity has continued to increase over the last 10 years, resulting in lifestyle changes. Obesity has become a serious national problem and is no longer a concern only in Western countries $[28,29]$. Considering that these issues remain a concern and that obesity is an important factor affecting mental health, it is necessary to design effective strategies to prevent and manage suicidal ideation among the overweight and obese populations.

We found that weight control failure was significantly associated with suicidal ideation among obese females, whereas this association was not significant in obese males or overweight populations after multivariable adjustment. This finding can be explained by weight stigma. Weight stigma has been described as negative weight-related attitudes and beliefs. Obese individuals are often highly stigmatized [30], and obese females experience weight stigma more than do obese males [31, 32]. Weight stigma experiences were significantly related to depressive symptoms, decreased self-esteem and suicidality [33, 34]. Obese females in particular may be more vulnerable to the societal standards of beauty and obesity stigma compared with males, thereby degrading mental health. Hence, weight control failure among obese females may affect experience with weight stigma, thereby deteriorating psychological wellbeing, and in particular, suicidal ideation.

Our findings suggests that efforts to reduce the high suicide rate should target obese females. Considering that the prevalence of obesity is high among middleaged women and that the age-specific obesity rate in Korean females has recently increased sharply, obese females are an important target group for intervention [35]. Despite its issues remain a concern, programs to resolve issues are rare. From our findings, therefore, we suggest that suicide prevention programs need to focus on supporting obese females, such as encouraging physical activity, and supporting enrollment in weight control programs. In Korea, most weight control programs for obesity are focused on children. Despite positive outcomes, the few programs focusing on obese females experienced limitations such as barriers in the recruitment and retention of participants [36]. Therefore, policy makers should develop strategies for participants' continuous participation using effectiveness tools, such as telephone counseling and mobile phone SMS messages [37]. Such support can improve not only the mental but also the physical health of obese females.

In addition, our subgroup analysis indicated that menopause, household composition, or low income potentially 
Table 3 Results of multivariable logistic regression analysis for the association between weight control failure and suicidal ideation

\begin{tabular}{|c|c|c|c|c|c|c|c|c|c|c|c|c|c|c|c|c|}
\hline \multirow{4}{*}{$\begin{array}{l}\text { Variables } \\
\text { Weight control failure }\end{array}$} & \multicolumn{8}{|c|}{ Suicidal ideation (Male) } & \multicolumn{8}{|c|}{ Suicidal ideation (Female) } \\
\hline & \multicolumn{4}{|c|}{ BMI $23 \sim 24.9$} & \multicolumn{4}{|c|}{$\mathrm{BMI} \geq 25$} & \multicolumn{4}{|c|}{ BMI $23 \sim 24.9$} & \multicolumn{4}{|c|}{$\mathrm{BMI} \geq 25$} \\
\hline & \multirow[t]{2}{*}{$a-O R^{a}$} & \multicolumn{3}{|c|}{$95 \% \mathrm{Cl}$} & \multirow[t]{2}{*}{$a-O R^{a}$} & \multicolumn{3}{|c|}{$95 \% \mathrm{Cl}$} & \multirow[t]{2}{*}{$a-O R^{a}$} & \multicolumn{3}{|c|}{$95 \% \mathrm{Cl}$} & \multirow[t]{2}{*}{$\overline{a-O R^{a}}$} & \multicolumn{3}{|c|}{$95 \% \mathrm{Cl}$} \\
\hline & & & & & & & & & & & & & & & & \\
\hline No & 1.00 & - & & - & 1.00 & - & & - & 1.00 & - & & - & 1.00 & - & & - \\
\hline Yes & 0.77 & 0.33 & - & 1.80 & 1.54 & 0.91 & - & 2.60 & 1.09 & 0.70 & - & 1.18 & 1.70 & 1.21 & - & 2.39 \\
\hline \multicolumn{17}{|l|}{ Age } \\
\hline $40 \sim 49$ & 1.00 & - & & - & 1.00 & - & & - & 1.00 & - & & - & 1.00 & - & & - \\
\hline $50 \sim 59$ & 1.37 & 0.64 & - & 2.94 & 0.99 & 0.52 & - & 1.86 & 1.19 & 0.68 & - & 2.10 & 0.70 & 0.42 & - & 1.17 \\
\hline $60 \sim 69$ & 1.13 & 0.42 & - & 3.03 & 1.28 & 0.54 & - & 3.04 & 1.27 & 0.57 & - & 2.83 & 1.14 & 0.61 & - & 2.14 \\
\hline$\geq 70$ & 2.27 & 0.67 & - & 7.75 & 1.45 & 0.59 & - & 3.60 & 1.16 & 0.37 & - & 3.59 & 1.43 & 0.67 & - & 3.05 \\
\hline \multicolumn{17}{|l|}{ Income } \\
\hline High & 1.00 & & - & & 1.00 & & - & & 1.00 & & - & & 1.00 & & - & \\
\hline Middle & 0.95 & 0.46 & - & 1.93 & 1.02 & 0.61 & - & 1.71 & 0.86 & 0.52 & - & 1.43 & 1.57 & 1.05 & - & 2.35 \\
\hline Low & 1.52 & 0.63 & - & 3.69 & 1.79 & 1.02 & - & 3.14 & 1.57 & 0.86 & - & 2.89 & 2.12 & 1.35 & - & 3.32 \\
\hline \multicolumn{17}{|l|}{ Education level } \\
\hline Less than middle school & 1.00 & - & & - & 1.00 & - & & - & 1.00 & - & & - & 1.00 & - & & - \\
\hline High school graduate & 0.81 & 0.37 & - & 1.75 & 0.56 & 0.30 & - & 1.02 & 0.77 & 0.47 & - & 1.26 & 0.72 & 0.48 & - & 1.08 \\
\hline University graduate & 0.88 & 0.38 & - & 2.01 & 0.55 & 0.29 & - & 1.07 & 0.60 & 0.30 & - & 1.22 & 0.75 & 0.42 & - & 1.35 \\
\hline \multicolumn{17}{|l|}{ Economic activity } \\
\hline Employed & 1.00 & - & & - & 1.00 & - & & - & 1.00 & - & & - & 1.00 & - & & - \\
\hline Unemployed & 1.23 & 0.64 & - & 2.41 & 1.18 & 0.61 & - & 2.27 & 1.29 & 0.83 & - & 2.01 & 0.95 & 0.70 & - & 1.31 \\
\hline Marital status & & & & & & & & & & & & & & & & \\
\hline Married & 1.00 & - & & - & 1.00 & - & & - & 1.00 & - & & - & 1.00 & - & & - \\
\hline Single & 0.51 & 0.08 & - & 3.36 & 3.06 & 1.00 & - & 9.34 & 2.00 & 0.52 & - & 7.66 & 0.30 & 0.04 & - & 1.99 \\
\hline Household composition & & & & & & & & & & & & & & & & \\
\hline Two Generation & 1.00 & - & & - & 1.00 & - & & - & 1.00 & - & & - & 1.00 & - & & - \\
\hline First Generation & 0.94 & 0.45 & - & 1.99 & 0.84 & 0.45 & - & 1.56 & 1.64 & 0.97 & - & 2.80 & 0.93 & 0.63 & - & 1.39 \\
\hline Three Generation & 1.33 & 0.58 & - & 3.05 & 0.56 & 0.25 & - & 1.28 & 0.87 & 0.45 & - & 1.70 & 1.22 & 0.79 & - & 1.89 \\
\hline Alcohol intake & & & & & & & & & & & & & & & & \\
\hline No & 1.00 & - & & - & 1.00 & - & & - & 1.00 & - & & - & 1.00 & - & & - \\
\hline Yes & 1.17 & 0.56 & - & 2.46 & 0.84 & 0.45 & - & 1.56 & 0.95 & 0.59 & - & 1.52 & 1.08 & 0.73 & - & 1.60 \\
\hline Stress awareness & & & & & & & & & & & & & & & & \\
\hline Low & 1.00 & - & & - & 1.00 & - & & - & 1.00 & - & & - & 1.00 & - & & - \\
\hline High & 3.93 & 2.24 & - & 6.90 & 3.79 & 2.14 & - & 4.42 & 4.78 & 3.09 & - & 7.40 & 3.35 & 2.45 & - & 4.59 \\
\hline Depression mood & & & & & & & & & & & & & & & & \\
\hline No & 1.00 & - & & - & 1.00 & - & & - & 1.00 & - & & - & 1.00 & - & & - \\
\hline Yes & 15.08 & 8.20 & - & 27.75 & 10.35 & 6.35 & - & 16.84 & 6.23 & 4.10 & - & 9.49 & 6.86 & 4.91 & - & 9.59 \\
\hline Moderate physical activity & & & & & & & & & & & & & & & & \\
\hline No & 1.00 & - & & - & 1.00 & - & & - & 1.00 & - & & - & 1.00 & - & & - \\
\hline Yes & 1.28 & 0.55 & - & 1.47 & 0.90 & 0.55 & - & 1.47 & 1.04 & 0.69 & - & 1.56 & 0.88 & 0.63 & - & 1.21 \\
\hline Subjective body perception & & & & & & & & & & & & & & & & \\
\hline Slim \& Normal & 1.00 & - & & - & 1.00 & - & & - & 1.00 & - & & - & 1.00 & - & & - \\
\hline Fat & 1.49 & 0.56 & - & 2.46 & 0.71 & 0.38 & - & 1.30 & 0.97 & 0.62 & - & 1.50 & 0.89 & 0.54 & - & 1.46 \\
\hline
\end{tabular}


Table 3 Results of multivariable logistic regression analysis for the association between weight control failure and suicidal ideation (Continued)

\begin{tabular}{|c|c|c|c|c|c|c|c|c|c|c|c|c|c|c|c|c|}
\hline \multicolumn{17}{|c|}{ Perceive health status } \\
\hline Healthy & 1.00 & - & & - & 1.00 & - & & - & 1.00 & - & & - & 1.00 & - & & - \\
\hline Unhealthy & 1.29 & 0.71 & - & 2.37 & 3.14 & 1.92 & - & 5.13 & 2.29 & 1.42 & - & 3.71 & 1.38 & 1.00 & - & 1.92 \\
\hline \multicolumn{17}{|l|}{ Menopause } \\
\hline Not yet & - & & - & & - & & - & & 1.00 & - & & - & 1.00 & - & & - \\
\hline Yes & - & & - & & - & & - & & 1.24 & 0.73 & - & 2.11 & 1.65 & 1.02 & - & 2.65 \\
\hline \multicolumn{17}{|l|}{ Year } \\
\hline 2008 & 1.00 & & & & 1.00 & & & & 1.00 & - & & - & 1.00 & - & & - \\
\hline 2009 & 1.35 & 0.54 & - & 3.33 & 0.39 & 0.20 & - & 0.77 & 0.70 & 0.35 & - & 1.40 & 0.90 & 0.58 & - & 1.41 \\
\hline 2010 & 0.95 & 0.40 & - & 2.26 & 0.63 & 0.33 & - & 1.22 & 1.14 & 0.61 & - & 2.13 & 0.91 & 0.55 & - & 1.52 \\
\hline 2011 & 1.31 & 0.50 & - & 3.41 & 1.20 & 0.67 & - & 2.17 & 0.97 & 0.51 & - & 1.84 & 0.51 & 0.31 & - & 0.83 \\
\hline 2012 & 1.25 & 0.47 & - & 3.31 & 0.86 & 0.41 & - & 1.82 & 0.84 & 0.43 & - & 1.64 & 0.64 & 0.39 & - & 1.08 \\
\hline Hosmer-Leme & & \multicolumn{4}{|c|}{$P=0.4263$} & \multicolumn{4}{|c|}{$P=0.3487$} & \multicolumn{4}{|c|}{$P=0.3573$} & \multicolumn{3}{|c|}{$P=0.4730$} \\
\hline
\end{tabular}

Notes: ${ }^{a}$ a-OR adjusted by weight control failure, age, income, education level, economic activity, marital status, household composition, alcohol intake, stress awareness, depression mood, moderate physical activity, subjective body perception, perceive health status, menopause, year

affect the association between weight control failure and suicidal ideation, even though the modifying effect was not significant. The overall trends seen among our findings have serious implications for the management of suicidal ideation. Several studies offer potential explanations. Regarding menopause, females may experience weight gain during the peri-menopausal to postmenopausal period, and this weight gain may exacerbate the changes in health risk factors that appear during menopause [20,38]. For that reasons, obese females may experience the weight gain. Thus, policy makers should consider health policy providing hormone injections for women experiencing menopause. Regarding household composition, previous studies reported that family members and support networks including parents, spouses, and friends can increase the effectiveness of weight control in obese populations. Such support networks would be most effective in eradicating negative attitudes and bias regarding weight $[39,40]$. Therefore, policy makers should consider programs utilizing family and a support network, it could help weight control in obese populations.

Meanwhile, we found a gradient in suicidal ideation by socio-demographic factors such as education, income, and employment status in both males and females. More disadvantage males and females were more likely to report suicidal ideation, in particular obese females. Few studies offer potential explanations by focusing relationship between socioeconomic gradient and obesity. Which relationship was well established through previous studies [41, 42]. For example, because higher socioeconomic groups tend to have a healthier diet, higher educational attainment, income and occupational status were associated with lower risk of obesity. In addition, studies suggests that the relation between socio-economic inequality and obesity is stronger among females than among males $[43,44]$. On the other hands, obese females are socially and economically disadvantaged [45]. They are less likely to practice the healthy dieting thereby increasing the weight. Therefore, weight gain and unhealthy dietary practices due to socio and economic disadvantage in women may lead to more weight control failure and weight stigma, thereby exacerbating the mental health. Meanwhile, few studies has shown an inverse results between socioeconomic status and obesity [46, 47]. These studies found that high education attainment or high income was related to prevalence of obesity in females. Based our results and previous studies, the relationship between social gradient and suicidal ideation among obese population are not currently, because studies on those relationship is less well established. Therefore, further studies on those findings are needed.

This study has several strengths compared with previous studies. First, the study used a large representative sample, and data were collected from a nationally representative population. Second, to our knowledge, our study is the first to report on the relationship between weight control failure and suicidal ideation among obese populations. Previous studies focused only on the relationship between weight-based stigmatization or obesity and mental health in adolescents. Finally, our study focused on the cause of suicidal ideation among overweight and obese populations.

However, there were also several limitations. First, the present study was unable to identify a causal relationship between weight control failure and suicidal ideation, because the study design was cross sectional, and information was obtained via self-report. Second, our subgroup analysis findings are limited with regard to the interpretation of the results, because the modifying effect was not significant, 
Table 4 Results of subgroup analysis for the relationship between weight control failure and suicidal ideation by income, household composition, or menopause

\begin{tabular}{|c|c|c|c|c|c|c|c|c|c|c|c|c|c|c|c|c|c|}
\hline \multirow[t]{3}{*}{ Variables } & & \multicolumn{8}{|l|}{ Male } & \multicolumn{8}{|c|}{ Female } \\
\hline & & \multicolumn{4}{|c|}{ BMI $23 \sim 24.9$} & \multicolumn{4}{|c|}{$B M I \geq 25$} & \multicolumn{4}{|c|}{ BMI $23 \sim 24.9$} & \multicolumn{4}{|c|}{$\mathrm{BMI} \geq 25$} \\
\hline & & \multirow[t]{2}{*}{$a-O R^{a}$} & \multicolumn{3}{|c|}{$95 \% \mathrm{Cl}$} & $a-O R^{a}$ & \multicolumn{3}{|c|}{$95 \% \mathrm{Cl}$} & $a-O R^{a}$ & \multicolumn{3}{|c|}{$95 \% \mathrm{Cl}$} & $\mathrm{a}-O R^{a}$ & \multicolumn{3}{|c|}{$95 \% \mathrm{Cl}$} \\
\hline & & & & & & & & & & & & & & & & & \\
\hline \multirow[t]{2}{*}{ High } & No & 1.00 & & & & 1.00 & & & & 1.00 & & & & 1.00 & & & \\
\hline & Yes & 0.33 & 0.07 & - & 1.54 & 0.52 & 0.13 & - & 2.11 & 0.80 & 0.31 & - & 2.03 & 1.65 & 0.65 & - & 4.24 \\
\hline \multirow[t]{2}{*}{ Middle } & No & 1.00 & & & & 1.00 & & & & & & & & 1.00 & & & \\
\hline & Yes & 1.86 & 0.69 & - & 5.03 & 1.37 & 0.66 & - & 2.88 & 1.17 & 0.61 & - & 2.23 & 1.67 & 1.08 & - & 2.60 \\
\hline \multirow[t]{2}{*}{ Low } & No & 1.00 & & & & 1.00 & & & & & & & & 1.00 & & & \\
\hline & Yes & 0.22 & 0.02 & - & 2.74 & 2.27 & 0.89 & - & 5.81 & 1.10 & 0.42 & - & 2.90 & 1.87 & 1.00 & - & 3.72 \\
\hline Household composition & Weight control failure & & & & & & & & & & & & & & & & \\
\hline \multirow[t]{2}{*}{ One generation } & No & 1.00 & & & & 1.00 & & & & 1.00 & & & & 1.00 & & & \\
\hline & Yes & 0.63 & 0.13 & - & 3.03 & 0.62 & 0.20 & - & 1.95 & 0.74 & 0.28 & - & 1.99 & 1.88 & 1.08 & - & 3.25 \\
\hline \multirow[t]{2}{*}{ Two generation } & No & 1.00 & & & & 1.00 & & & & 1.00 & & & & 1.00 & & & \\
\hline & Yes & 1.29 & 0.52 & - & 3.17 & 2.46 & 1.32 & - & 4.57 & 1.19 & 0.66 & - & 2.13 & 1.64 & 1.02 & - & 2.62 \\
\hline \multirow[t]{2}{*}{ Three generation } & No & 1.00 & & & & 1.00 & & & & 1.00 & & & & 1.00 & & & \\
\hline & Yes & 0.12 & 0.01 & - & 1.74 & 2.26 & 0.29 & - & 17.60 & 2.01 & 0.22 & - & 18.1 & 1.05 & 0.42 & - & 2.62 \\
\hline Menopause & Weight control failure & & & & & & & & & & & & & & & & \\
\hline \multirow[t]{2}{*}{ Not yet } & No & - & - & & - & - & - & & - & 1.00 & & & & 1.00 & & & \\
\hline & Yes & - & - & & - & - & - & & - & 1.31 & 0.68 & - & 2.53 & 1.58 & 0.90 & - & 2.80 \\
\hline \multirow[t]{2}{*}{ Yes } & No & - & - & & - & - & - & & - & 1.00 & & & & 1.00 & & & \\
\hline & Yes & - & - & & - & - & - & & - & 0.91 & 0.47 & - & 1.76 & 1.80 & 1.18 & - & 2.75 \\
\hline
\end{tabular}

Notes: a a-OR adjusted by weight control failure, age, income, education level, economic activity, marital status, household composition, alcohol intake, stress awareness, depression mood, moderate physical activity, subjective body perception, perceive health status, menopause, year

although the findings showed significant differences in each group. Therefore, other studies are needed to confirm our findings. Third, we did not investigate the reasons behind weight control failure and change in weight. Furthermore, we did not consider various factors related to weight change, such as food intake volume, exercise frequency and exercise intensity. Fourth, we could not accurately measure suicidal ideation, because the question pertaining to suicidal ideation required only a "yes" or "no" answer. In addition, we could not accurately assess weight control failure, because the answers were subjective. Therefore, self-reporting of the respondents could have led to an underestimation of the actual relationship between weight control failure and suicidal ideation.

Despite the limitations, this is the first study to investigate the association between weight control failure and suicidal ideation among obese and overweight Koreans. Considering that the high prevalence of suicide and the increasing prevalence of obesity in Korea, our findings are important for health policy makers to identify solutions for controlling suicide problem.

\section{Conclusion}

In conclusion, weight control failure had an effect on suicidal ideation. Suicidal ideation in obese females was significantly higher than that in other populations. Our findings suggest a need to focus on suicidal ideation in middle-aged and older obese females in particular.

\section{Competing interests}

The authors declare that they have no competing interests.

\section{Authors' contributions}

YJ managed and analyzed data and wrote the manuscript. YJ and KT contributed to analysis the data and manuscript writing. $T H, W R$, and $J H$ provided intellectual input for the development of the manuscript. EC designed and supervised the present study. All member contributed to the implementation and quality assurance of the study. All authors read and approved the final manuscript.

\section{Acknowledgement}

We thank Department of Health and Human Services Centers for Disease Control and Prevention that provided meaningful data.

\section{Author details}

'Department of Public Health, Graduate School, Yonsei University, Seoul, Republic of Korea. ${ }^{2}$ Institute of Health Services Research, Yonsei University College of Medicine, Seoul, Republic of Korea. ${ }^{3}$ Department of Preventive Medicine, Yonsei University College of Medicine, Seoul, Republic of Korea. 
${ }^{4}$ Department of Health Administration and Management, College of Medical Science, Soonchunhyang University, Asan, Republic of Korea. ${ }^{5}$ Department of Preventive Medicine \& Institute of Health Services Research, Yonsei University College of Medicine, 50 Yonsei-ro, Seodaemun-gu, Seoul 120-752, Korea.

\section{Received: 27 October 2015 Accepted: 8 March 2016}

\section{Published online: 15 March 2016}

\section{References}

1. Jang S-I, Nam J-M, Choi J, Park E-C. Disease management index of potential years of life lost as a tool for setting priorities in national disease control using OECD health data. Health Policy. 2014;115(1):92-9.

2. Hewlett E, Moran V. Making Mental Health Count: The Social and Economic Costs of Neglecting Mental Health Care. In: OECD Health Policy Studies. Paris: OECD Publishing; 2014.

3. Kessler RC, Borges G, Walters EE. Prevalence of and risk factors for lifetime suicide attempts in the National Comorbidity Survey. Arch Gen Psychiatry. 1999;56(7):617-26.

4. Jeon G-S, Jang S-N, Rhee S-J, Kawachi I, Cho S-I. Gender differences in correlates of mental health among elderly Koreans. J Gerontol Ser B Psychol Sci Soc Sci. 2007;62(5):S323-9.

5. Kim K, Ryu E, Chon M-Y, Yeun E-J, Choi S-Y, Seo J-S, Nam B-W. Internet addiction in Korean adolescents and its relation to depression and suicidal ideation: a questionnaire survey. Int J Nurs Stud. 2006;43(2):185-92.

6. Kim D-S, Kim H-S. Early initiation of alcohol drinking, cigarette smoking, and sexual intercourse linked to suicidal ideation and attempts: findings from the 2006 Korean Youth Risk Behavior Survey. Yonsei Med J. 2010;51(1):18-26.

7. Diefenbach GJ, Woolley SB, Goethe JW. The association between self-reported anxiety symptoms and suicidality. J Nerv Ment Dis. 2009;197(2):92-7.

8. Wardle J, Cooke L. The impact of obesity on psychological well-being. Best Pract Res Clin Endocrinol Metab. 2005;19(3):421-40.

9. Blaine B. Does depression cause obesity? A meta-analysis of longitudinal studies of depression and weight control. J Health Psychol. 2008;13(8):1190-7.

10. Fabricatore AN, Wadden TA. Psychological aspects of obesity. Clin Dermatol. 2004;22(4):332-7.

11. Reilly JJ, Methven E, McDowell ZC, Hacking B, Alexander D, Stewart L, Kelnar CJ. Health consequences of obesity. Arch Dis Child. 2003;88(9):748-52.

12. Pi-Sunyer FX. Medical hazards of obesity. Ann Intern Med. 1993;119(7_Part_2): 655-60.

13. Davidson MH, Hauptman J, DiGirolamo M, Foreyt JP, Halsted CH, Heber D, Heimburger DC, Lucas CP, Robbins DC, Chung J. Weight control and risk factor reduction in obese subjects treated for 2 years with orlistat: a randomized controlled trial. JAMA. 1999;281(3):235-42.

14. Neumark-Sztainer D, Story M, Resnick MD, Blum RW. Psychosocial concerns and weight control behaviors among overweight and nonoverweight Native American adolescents. J Am Diet Assoc. 1997;97(6):598-604.

15. Falkner NH, Neumark-Sztainer D, Story M, Jeffery RW, Beuhring T, Resnick MD. Social, educational, and psychological correlates of weight status in adolescents. Obes Res. 2001;9(1):32-42.

16. Crow S, Eisenberg ME, Story M, Neumark-Sztainer D. Suicidal behavior in adolescents: relationship to weight status, weight control behaviors, and body dissatisfaction. Int J Eat Disord. 2008;41(1):82-7.

17. Park IB, Baik SH. Epidemiologic characteristics of diabetes mellitus in Korea: current status of diabetic patients using Korean Health Insurance Database. Korean Diab J. 2009:33(5):357-62.

18. Forman-Hoffman VL, Yankey JW, Hillis SL, Wallace RB, Wolinsky FD. Weight and depressive symptoms in older adults: direction of influence? I Gerontol Ser B Psychol Sci Soc Sci. 2007;62(1):S43-51.

19. Hong JS, Yi S-W, Kang HC, Jee SH, Kang HG, Bayasgalan G, Ohrr H. Age at menopause and cause-specific mortality in South Korean women: Kangwha Cohort Study. Maturitas. 2007:56(4):411-9.

20. Eliassen AH, Colditz GA, Rosner B, Willett WC, Hankinson SE. Adult weight change and risk of postmenopausal breast cancer. JAMA. 2006;296(2):193-201.

21. Simon RW. Gender, multiple roles, role meaning, and mental health. J Health Soc Behav. 1995;36:182-94

22. Chin YR, Lee HY, So ES. Suicidal ideation and associated factors by sex in Korean adults: a population-based cross-sectional survey. Int J Public Health. 2011:56(4):429-39.

23. Lee M-S, Shin J-S, Lee J, Lee YJ, Kim M-R, Park KB, Shin D, Cho J-H, Ha I-H. The association between mental health, chronic disease and sleep duration in Koreans: a cross-sectional study. BMC Public Health. 2015;15(1):1.
24. EC WHO. Appropriate body-mass index for Asian populations and its implications for policy and intervention strategies. Lancet. 2004;363(9403): 157.

25. Kweon S, Kim Y, Jang M-J, Kim Y, Kim K, Choi S, Chun C, Khang Y-H, Oh K Data resource profile: the Korea National Health and Nutrition Examination Survey (KNHANES). Int J Epidemiol. 2014;43(1):69-77.

26. Hosmer Jr DW, Lemeshow S, Sturdivant RX: Interpretation of the Fitted Logistic Regression Model. Applied Logistic Regression, Third Edition 2013:49-88. John Wiley \& Sons, Inc., Hoboken, NJ, USA. doi: 10.1002/9781118548387.ch3.

27. Statistics Korea. Annual report on the cause of death statistics. Seioul: Korean National statistical Office; 2014

28. Park H, Park C, Oh S, Yoo H. Prevalence of obesity and metabolic syndrome in Korean adults. Obes Rev. 2008;9(2):104-7.

29. Kim DM, Ahn CW, Nam SY. Prevalence of obesity in Korea. Obes Rev. 2005; 6(2):117-21.

30. Puhl R, Brownell KD. Bias, discrimination, and obesity. Obes Res. 2001;9(12): 788-805.

31. Cash TF, Brown TA. Gender and body images: Stereotypes and realities. Sex Roles. 1989;21(5-6):361-73.

32. Pingitore R, Dugoni BL, Tindale RS, Spring B. Bias against overweight job applicants in a simulated employment interview. J Appl Psychol. 1994;79(6): 909.

33. Puhl RM, Heuer CA. The stigma of obesity: a review and update. Obesity. 2009;17(5):941-64.

34. Friedman KE, Reichmann SK, Costanzo PR, Zelli A, Ashmore JA, Musante GJ. Weight stigmatization and ideological beliefs: relation to psychological functioning in obese adults. Obes Res. 2005;13(5):907-16.

35. Health $\mathrm{KMO}$, Welfare. Korea national health and nutrition survey in 2008. Seoul: Korea Ministry of Health and Welfare; 2009.

36. Sim JH, Go DG, Yoo YJ. The effect of an obesity control program on body composition and blood lipid level among middle-aged obese women in a health center. J Korean Acad Community Health Nurs. 2007;18(2):201-10.

37. Newman VA, Flatt SW, Pierce JP. Telephone counseling promotes dietary change in healthy adults: results of a pilot trial. J Am Diet Assoc. 2008; 108(8):1350-4.

38. Wing RR, Matthews KA, Kuller LH, Meilahn EN, Plantinga PL. Weight gain at the time of menopause. Arch Intern Med. 1991:151(1):97-102.

39. Wing RR, Jeffery RW. Benefits of recruiting participants with friends and increasing social support for weight loss and maintenance. J Consult Clin Psychol. 1999;67(1):132.

40. McLean N, Griffin S, Toney K, Hardeman W. Family involvement in weight control, weight maintenance and weight-loss interventions: a systematic review of randomised trials. Int J Obes. 2003;27(9):987-1005.

41. McLaren L. Socioeconomic status and obesity. Epidemiol Rev. 2007;29(1):29-48,

42. Pickett KE, Kelly S, Brunner E, Lobstein T, Wilkinson RG. Wider income gaps, wider waistbands? An ecological study of obesity and income inequality. J Epidemiol Community Health. 2005;59(8):670-4.

43. Sarlio-Lähteenkorva S, Lahelma E. The association of body mass index with social and economic disadvantage in women and men. Int J Epidemiol. 1999;28(3):445-9.

44. Molarius A, Seidell JC, Sans S, Tuomilehto J, Kuulasmaa K. Educational level, relative body weight, and changes in their association over 10 years: an international perspective from the WHO MONICA Project. Am J Public Health. 2000;90(8):1260-8.

45. Zhang Q, Wang Y. Socioeconomic inequality of obesity in the United States: do gender, age, and ethnicity matter? Soc Sci Med. 2004;58(6):1171-80.

46. Mendez M, Cooper R, Luke A, Wilks R, Bennett F, Forrester T. Higher income is more strongly associated with obesity than with obesity-related metabolic disorders in Jamaican adults. Int J Obes. 2004:28(4):543-50.

47. Ferrer CP, McMunn A, Dommarco JAR, Brunner EJ: Educational inequalities in obesity among Mexican women: time-trends from 1988 to 2012. PloS one 2014, 9(3):e90195. doi: 10.1371/journal.pone.0090195. 